\title{
On the Binomial Confidence Interval and Probabilistic Robust Control
}

\author{
Xinjia Chen, Kemin Zhou and Jorge L. Aravena \\ Department of Electrical and Computer Engineering \\ Louisiana State University \\ Baton Rouge, LA 70803
}

Received in July 2002, Revised in February 2004

\begin{abstract}
The Clopper-Pearson confidence interval has ever been documented as an exact approach in some statistics literature. More recently, such approach of interval estimation has been introduced to probabilistic control theory and has been referred as non-conservative in control community. In this note, we clarify the fact that the so-called exact approach is actually conservative. In particular, we derive analytic results demonstrating the extent of conservatism in the context of probabilistic robustness analysis. This investigation encourages seeking better methods of confidence interval construction for robust control purpose.
\end{abstract}

\section{Introduction}

Ever since Stengel and Ray originated the concept of stochastic robustness, there has been growing interest in developing probabilistic methods for robust control. Significant contributions have been made by a number of researchers (see, e.g., [1, 2, 3, 4, 15], [10, 11, 12, 13, 14, 15, 16, 17] and the reference therein). A fundamental problem in the area of probabilistic robustness analysis is to estimate the probability that a certain robustness requirement is guaranteed for an uncertain dynamic system. The estimation of such probability relies essentially on Monte Carlo simulation. When an estimate of the probability is obtained from i.i.d. observations of fixed sample size, an important concern is how accurate this estimate is. To be useful, a numerical method must include a basis for error assessment. The Monte Carlo method is no exception. Stengel and Ray [12, 14] first introduced the Clopper-Pearson confidence interval [6] to evaluate the accuracy of estimation in the context of robustness analysis. In their works, such approach has been considered as non-conservative.

In this note, we would like to clarify the fact that the Clopper-Pearson confidence interval is conservative. The erroneous understanding of the confidence interval is not due to researchers in 
control area. Historically, such erroneous concept can be traced back to some statistical literature. The Clopper-Pearson confidence interval was usually referred as the "exact" confidence interval. It has been documented as a non-conservative approach in some statistics literature (see, for example, Page 697-698 of [9] and Page 95-103 of [8]). Although the so-called exact confidence interval was proposed by Clopper and Pearson in 1934, its rigorous probabilistic implication was not made clear until 1958 by Cluniess-Ross [7. Interestingly, it was proved in [7] that such "exact" approach is actually conservative.

Since the confidence coefficient is directly related to the risk and safety of control systems in the context of probabilistic design and analysis, a clear understanding of the conservatism of confidence interval construction will help making the tradeoff between the risk and performance enhancement. To this purpose, we investigate the conservatism of the confidence interval. We obtain analytic results indicating that, in the scenario of rare events (especially in the context of probabilistic robustness analysis), the conservatism is not trivial and better methods of confidence construction should be sought.

\section{Binomial Confidence Interval}

Let the probability space be denoted as $(\Omega, F, P)$ where $\Omega, F, P$ are the sample space, the algebra of events and the probability measure respectively. Let $X$ be a Bernoulli random variable with distribution $\operatorname{Pr}\{X=1\}=\mathbb{P}_{X}, \quad \operatorname{Pr}\{X=0\}=1-\mathbb{P}_{X}$ where $\mathbb{P}_{X} \in(0,1)$. Let the sample size $N$ and confidence parameter $\delta \in(0,1)$ be fixed. We refer an observation with value 1 as a successful trial. Let $K$ denote the number of successful trials during the $N$ i.i.d. sampling experiments. Let $k=K(\omega)$ where $\omega$ is a sample point in the sample space $\Omega$.

The classic Clopper-Pearson lower confidence limit $L_{N, k, \delta}$ and upper confidence limit $U_{N, k, \delta}$ are given respectively by

$$
L_{N, k, \delta}:= \begin{cases}0 & \text { if } k=0 \\ \underline{p} & \text { if } k>0\end{cases}
$$

and

$$
U_{N, k, \delta}:= \begin{cases}1 & \text { if } k=N \\ \bar{p} & \text { if } k<N\end{cases}
$$

where $\underline{p} \in(0,1)$ is the solution of equation $\sum_{j=0}^{k-1}\left(\begin{array}{c}N \\ j\end{array}\right) \underline{p}^{j}(1-\underline{p})^{N-j}=1-\frac{\delta}{2}$ and $\bar{p} \in(0,1)$ is the solution of equation $\sum_{j=0}^{k}\left(\begin{array}{c}N \\ j\end{array}\right) \bar{p}^{j}(1-\bar{p})^{N-j}=\frac{\delta}{2}$. Define random variable $L: \Omega \rightarrow[0,1]$ by $L(\omega):=$ $L_{N, K(\omega), \delta} \quad \forall \omega \in \Omega$ and random variable $U: \Omega \rightarrow[0,1]$ by $U(\omega):=U_{N, K(\omega), \delta} \forall \omega \in \Omega$. Then the random interval $[L, U]$ is referred as the classic Clopper-Pearson confidence interval. Its probabilistic implication was quite often erroneously interpreted as

$$
\operatorname{Pr}\left\{L \leq \mathbb{P}_{X} \leq U\right\}=1-\delta
$$


or

$$
\operatorname{Pr}\left\{L<\mathbb{P}_{X}<U\right\}=1-\delta .
$$

However, it was proved by Cluniess-Ross [7] in 1958 that

$$
\operatorname{Pr}\left\{L \leq \mathbb{P}_{X} \leq U\right\}>1-\delta
$$

and

$$
\operatorname{Pr}\left\{L<\mathbb{P}_{X}<U\right\} \geq 1-\delta \text {. }
$$

These inequalities have been demonstrated by numerical experiments reported in the literature. For a better understanding of the conservatism, especially in the context of probabilistic robustness analysis, we shall investigate analytically how conservative the Clopper-Pearson interval can be.

\section{How Conservative?}

We refer the exact value of $\operatorname{Pr}\left\{L \leq \mathbb{P}_{X} \leq U\right\}$ as the coverage probability. We have the following results with regard to the conservatism of the Clopper-Pearson confidence interval.

Theorem 1 Let $N, \delta$ be fixed. If $\mathbb{P}_{X}$ or $1-\mathbb{P}_{X}$ is less than $1-\left(\frac{\delta}{2}\right)^{\frac{1}{N}}$, then the coverage probability will be at least $1-\frac{\delta}{2}$. Moreover, if $\left(\frac{\delta}{2}\right)^{\frac{1}{N}}<\mathbb{P}_{X}<1-\left(\frac{\delta}{2}\right)^{\frac{1}{N}}$, then the coverage probability is 1 .

Proof. For the simplicity of notation, define $\mathcal{S}(N, k, x):=\sum_{j=0}^{k}\left(\begin{array}{c}N \\ j\end{array}\right) x^{j}(1-x)^{N-j}$ for $x \in(0,1)$. Notice that $\mathbb{P}_{X} \geq\left(\frac{\delta}{2}\right)^{\frac{1}{N}}$ if and only if

$$
\mathcal{S}\left(N, N-1, \mathbb{P}_{X}\right)=1-\mathbb{P}_{X}^{N} \leq 1-\frac{\delta}{2}
$$

and observe that, for fixed $x \in(0,1), \mathcal{S}(N, k, x)$ increases monotonically with respect to $k$, we have that $\mathcal{S}\left(N, N-1, \mathbb{P}_{X}\right) \leq 1-\frac{\delta}{2}$ if and only if

$$
\mathcal{S}\left(N, k-1, \mathbb{P}_{X}\right) \leq 1-\frac{\delta}{2} \forall k \in\{1, \cdots, N\}
$$

By Lemma (3.8 a) on page 277 of [7], we have that $\mathcal{S}(N, k-1, x)$ decreases monotonically with respect to $x \in(0,1)$. Therefore, $L_{N, k, \delta}<\mathbb{P}_{X}$ for all $k \in\{1, \cdots, N\}$. Recall that $L_{N, N, \delta}=0<\mathbb{P}_{X}$, we thus have

$$
\mathbb{P}_{X} \geq\left(\frac{\delta}{2}\right)^{\frac{1}{N}} \Longleftrightarrow \operatorname{Pr}\left\{L \leq \mathbb{P}_{X}\right\}=1 .
$$

Similarly, we can show that

$$
\mathbb{P}_{X} \leq 1-\left(\frac{\delta}{2}\right)^{\frac{1}{N}} \Longleftrightarrow \operatorname{Pr}\left\{\mathbb{P}_{X} \leq U\right\}=1
$$




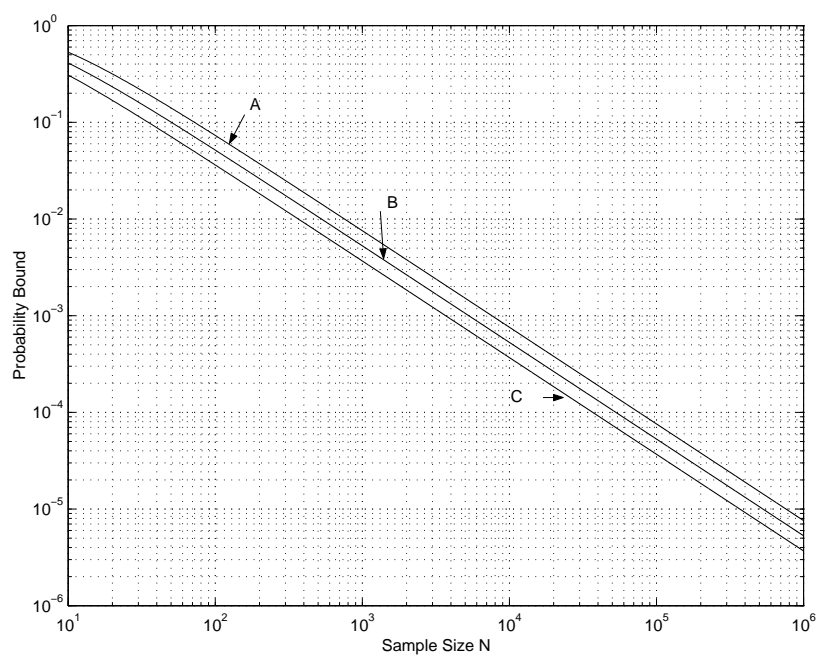

Figure 1: Conservatism of Clopper-Pearson Confidence Interval. Probability Bound $=1-\left(\frac{\delta}{2}\right)^{\frac{1}{N}}$. Plot A corresponds to $\delta=0.001$, plot $\mathrm{B}$ corresponds to $\delta=0.01$, plot $\mathrm{C}$ corresponds to $\delta=0.05$.

For the case that $\mathbb{P}_{X} \geq\left(\frac{\delta}{2}\right)^{\frac{1}{N}}$, by (1) and Bonferoni's inequality

$$
\begin{aligned}
\operatorname{Pr}\left\{L \leq \mathbb{P}_{X} \leq U\right\} & \geq \operatorname{Pr}\left\{L \leq \mathbb{P}_{X}\right\}+\operatorname{Pr}\left\{\mathbb{P}_{X} \leq U\right\}-1 \\
& =\operatorname{Pr}\left\{\mathbb{P}_{X} \leq U\right\} \\
& >1-\frac{\delta}{2} .
\end{aligned}
$$

Similarly, for the other case that $\mathbb{P}_{X} \leq 1-\left(\frac{\delta}{2}\right)^{\frac{1}{N}}$ we can show that $\operatorname{Pr}\left\{L \leq \mathbb{P}_{X} \leq U\right\}>1-\frac{\delta}{2}$. Thus the first statement is proved.

Finally, the proof of second statement is completed by making use of Bonferoni's inequality and inequalities (11) and (2).

Figure 1 shows bounds of binomial proportion for which the true coverage probability of the Clopper-Pearson confidence interval exceeds the prescribed confidence level by at least $\frac{\delta}{2}$. It can be seen that the conservatism is common in the scenario of studying rare events by the Monte Carlo method. For example, when constructing a 99\% confidence interval for the event of instability based on 10,000 Monte Carlo simulations, the true confidence level will be at least $99.5 \%$ if the probability of instability is smaller than 0.0005 (This number is the vertical coordinate of the point in plot B with horizontal coordinate 10,000). 


\section{Conclusion}

It is demonstrated that the Clopper-Pearson confidence interval is rather conservative for rare events.

The coverage probability can easily exceed the specified confidence level by at least $\frac{\delta}{2}$ and can be $100 \%$. Robustness issues such as instability and performance violation are normally interpreted as such rare events. Although the confidence parameter $\delta$ is usually a small number, the impact can be enormous due to its particular connection to the stability and performance of control systems in the probabilistic robust control framework. Our investigation suggests seeking better methods of confidence construction which are rigorous and less conservative.

\section{References}

[1] E. W. Bai, R. Tempo, and M. Fu. Worst-case properties of the uniform distribution and randomized algorithms for robustness analysis. Mathematics of Control, Signals and Systems, 11:183-196, 1998.

[2] B. R. Barmish and C. M. Lagoa. The uniform distribution: a rigorous justification for its use in robustness analysis. Mathematics of Control, Signals and Systems, 10:203-222, 1997.

[3] B. R. Barmish, C. M. Lagoa, and R. Tempo. Radially truncated uniform distributions for probabilistic robustness of control systems. Proc. of American Control Conference, pages 853857, June 1997.

[4] G. Calafiore, F. Dabbene, and R. Tempo. Randomized algorithms for probabilistic robustness with real and complex structured uncertainty. IEEE Transactions on Automatic Control, AC45:2218-2235, 2000.

[5] X. Chen, K. Zhou, and J. L. Aravena. Fast construction of robustness degradation function. SIAM Journal on Control and Optimization, 42:1960-1971, 2004.

[6] C. J. Clopper and E. S. Pearson. The use of confidence or fiducial limits illustrated in the case of the binomial. Biometrika, 26:404-413, 1934.

[7] C. W. Clunies-Ross. Interval estimation for the parameter of a binomial distribution. Biometrika, 45:275-279, 1958.

[8] W. J. Conover. Practical Nonparametric Statistics. John Wiley and Sons, 1977.

[9] A. Hald. Statistical Theory with Engineering Applications. John Wiley and Sons, 1952. 
[10] P. P. Khargonekar and A. Tikku. Randomized algorithms for robust control analysis and synthesis have polynomial complexity. Proceedings of IEEE Conference on Decision and Control, pages 3470-3475, December 1996.

[11] B. T. Polyak and P. S. Scherbakov. Random spherical uncertainty in estimation and robustness. IEEE Transactions on Automatic Control, AC-45:2145-2150, 2000.

[12] L. R. Ray and R. F. Stengel. A monte carlo approach to the analysis of control systems robustness. Automatica, 3:229-236, 1993.

[13] S. R. Ross and B. R. Barmish. Distributionally robust gain analysis for systems containing complexity. Proceedings of IEEE Conference on Decision and Control, pages 5020-5025, December 2001.

[14] R. F. Stengel and L. R. Ray. Stochastic robustness of linear time-invariant systems. IEEE Transactions on Automatic Control, AC-36:82-87, 1991.

[15] R. Tempo, E. W. Bai, and F. Dabbene. Probabilistic robustness analysis: explicit bounds for the minimum number of samples. Systems and Control Letters, 30:237-242, 1997.

[16] R. Tempo and F. Dabbene. Probabilistic robustness analysis and design of uncertain systems. In Progress in Systems and Control Theory (edited by G. Picci), pages 263-282. Birkhauser, 1999.

[17] M. Vidyasagar and V. D. Blondel. Probabilistic solutions to np-hard matrix problems. Automatica, 37:1597-1405, 2001. 